\title{
New Psychopedagogical Perspective for the National Education during the Albanian Renaissance
}

\author{
Esmeralda Dani \\ Department of Education, Luigj Gurakuqi University, Shkodër (Albania) \\ esmeraldahoti@yahoo.it
}

\section{Doi:10.5901/ajis.2015.v4n2p301}

Abstract

\begin{abstract}
The aim of this purpose is to give a panorama of the new perspective of educational system during the Albanian Renaissance (1878-1912), more specifically represented through the first pedagogical high school of Elbasan and the new criteria of the masses educational initiations. The main hypothesis of this study is that during this period, through the efforts of the Albanian patriots, it was possible to build the first foundations of national education and reappraise the Albanian's ideological and pedagogical education upon the European educational approaches. To test this hypothesis was evaluated the Albanian paper or textbooks published during this new historical and cultural period, compared with some studies carried out by Italian scholars of the same period. At the end it was demonstrated that the intellectual of Albanian Renaissance pursued a similar path to the Italian from the point of view of the ferment of ideas for school reforms only regarding the content of the proposals, since the initiative did not come, as in the case of the Italian reforms, from government but by the Albanian nationalists club, which perhaps was inspired by the reforms of the Italian government, since most of the Albanian intellectuals studied or worked with the arbëresh of Italy.
\end{abstract}

Keywords: Albanian Renaissance, National Education, the "Normale" of Elbasan, patriotic teachers.

\section{La Normale di Elbasan: il Maestro Come Educatore del Popolo}

Con una perspicace lungimiranza, i grandi patrioti albanesi furono abili a identificare la più emergente "penuria educativa" che prendeva vita nella nuova realtà storica ottomana: la costruzione di un'istruzione pedagogica nazionale (Osmani, 1998, 253). Nel 1908 Hil Mosi pubblicava l'articolo I nostri bisogni, in cui menzionava il problema attinente alla preparazione dei nuovi maestri come il più emergente da risolvere per il futuro del paese. Egli insisteva con perseveranza sulla pubblicazione dei testi scolastici e la sensibilizzazione di una nuova opinione pubblica sostenuta dai club nazionalisti e culturali come l'unica via per formare maestri albanesi capaci non solo ad applicare i metodi pedagogici ma anche di formare un degno cittadino per la propria patria. In queste circostanze, il pensiero per la costruzione di una scuola Normale diviene sempre più presente e reale, finché nel 1909 gli intellettuali di diverse associazioni culturali, dopo l'iniziativa del club di Salonicco si riunirono a Elbasan nel Congresso Nazionale per i problemi del paese, conosciuto anche come II Congresso delle scuole albanesi (Ibidem).

Visto l'attuale traguardo pedagogico, il sistema educativo albanese richiedeva una nuova riorganizzazione: l'istruzione e l'insegnamento dovevano essere tessute all'interno di un sistema indipendente, nel contenuto e nell'amministrazione, dal contesto ottomano; bisognava sormontare la condizione iniziale, cioè lo sviluppo solo dell'istruzione elementare, ed ergersi verticalmente verso scuole di più alto livello (Ibid., 255).

A settembre del 1909, a seguito all'eco prodotta dal congresso del 1908 di Elbasan sulla scelta dell'alfabeto albanese da adattare per il successivo sviluppo di una lingua nazionale standardizzata, gli attivisti speravano di provvedere alla risoluzione delle questioni 'scuola' e 'raccolta fondi' per sostenere questa nobile causa. Era comunque un terreno educativo aspro che poteva essere lavorato solo grazie all'apertura di una scuola media normale indirizzata alla formazione dei maestri elementari (Clayer, 2009, 558), la quale, nonostante ciò «non riuscì a consolidarsi definitivamente per un lungo periodo» (Ibid., 275).

Al principio del Novecento, la realtà del mondo magistrale e quella dell'istruzione elementare» costituirono un'importante prerogativa scientifica e didattica non solo per il fragile ridimensionamento ideologico e pedagogico dell'educazione albanese, ma anche per il più largo contesto europeo, e in particolar modo quello vicino alle dinamiche culturali albanesi, ovvero il contesto italiano, dato che «il mondo magistrale e l'istruzione elementare furono investite dall'insorgere di due vere e proprie emergenze nazionali, connesse la prima con l'accresciuto fabbisogno di maestri 
elementari, e la seconda con la ormai indilazionabile necessità di migliorare qualitativamente la loro formazione culturale e professionale (Sani, 2011, 392).

Nonostante tutto, accanto al problema della formazione di nuovi insegnanti e del perfezionamento della loro preparazione culturale e professionale, si poneva anche il miglioramento e il rinnovamento della formazione magistrale per riformare la scuola normale e il potenziamento del ruolo, delle funzioni, delle direzioni didattiche e della riforma dell'ispettorato scolastico. «L'accesso dei maestri alle carriere superiori, sotto questo profilo, si collegava strettamente al disegno di ridefinire in termini di competenza pedagogica e didattica - e non solo in termini di mera efficacia burocratica la funzione di ispezione e la stessa dirigenza scolastica» (Ibid., 394).

Gli uomini del Risorgimento albanese perseguivano un percorso simile a quello italiano dal punto di vista del fermento delle idee per la riforma della scuola normale soltanto per quando riguarda il contenuto delle proposte, siccome l'iniziativa non proveniva, come nel caso delle riforme italiane, dal governo ma dagli stessi club nazionalisti albanesi, i quali, avvertendo la necessità di profonde innovazioni pedagogiche e, forse ispirandosi alle riforme del governo italiano, giacché la maggior parte degli intellettuali albanesi studiava o collaborava con gli arbëresh d'Italia, decisero allora di aprire la prima scuola normale a Elbasan il'1 dicembre del 1909. La reazione della gente pare fosse entusiasmante, mentre famiglie di svariate zone del paese incalzavano a iscrivere i propri figli nella scuola. Anche se nei primi giorni della sua apertura lo staff pedagogico cominciò le lezioni soltanto con cinquanta alunni, entro il primo anno accademico la Normale di Elbasan, così battezzata, contava 160 allievi (Demiraj, Prifti, 2004, 129), dai quali cinquanta erano albanesi del Kosovo ("Normalisti", 1959, 19-20) organizzati da illustri patrioti come Hasan Prishtina, Bajram Curri, Nexhip Draga (Kumani, 1985, 3). Altre scuole furono istituite a Korça, Martanesh, Proger, Leskovik, ma anche in Kosovo come a Prishtina, Pejë e Gjakovë, dove svolgeva con successo la sua attività, il club di Scopie che era riuscito ad aprire nel 1909 anche una scuola privata di lingua albanese a Mitrovicë (Demiraj, Prifti, cit., 129-130).

L'apertura della Normale di Elbasan era anche un momento di soddisfazione dopo le lunghe battaglie dei patrioti albanesi, in particolar modo per Luigj Gurakuqi e Aleksandër Xhuvani (1880-1961), i due veri padri fondatori dell'istituzione. Xhuvani elenca le materie insegnate in questa scuola: «1. Matematica: aritmetica, geometria, algebra, trigonometria, astronomia ecc. 2. Fisica e Chimica; 3. Storia della natura, anatomia, fisiologia, zoologia, botanica, geologia, mineralogia; 4. Lingua albanese: scrittura, sintassi, letteratura, retorica, poetica; 5. Lingua turca. 6. Lingua francese; 7. Lingua inglese e greca a scelta; 8. Filosofia: psicologia, logica e pedagogia; 9. Religione: musulmana, cattolica e ortodossa; 10. Geografia; 11. Storia generale della Turchia e dell'Albania; 12. Disegno; 13. Musica; 14. Ginnastica» (Xhuvani, 1934, IV).

Dopo una produttiva collaborazione iniziata già nel 1906 a Napoli, finalmente essi erano riusciti ad aprire la scuola per i maestri elementari che, come diceva Gurakuqi nel giorno della sua apertura, «fu il desiderio e la speranza dei nostri padri da tanto tempo», anche se «in realtà noi non abbiamo mai avuto dei veri e prosperi pedagogisti» (Osmani, cit., 136).

Lo stesso discorso effettuato in Italia da Luigi Credaro sul ruolo del maestro come educatore del popolo e soprattutto sulla preparazione professionale, pedagogica, didattica e metodologica (Sani, ibid., 398), fu ripreso anche dagli intellettuali albanesi durante i lavori del congresso, il più importante di tutti Luigj Gurakuqi che, come sostiene Hoti, «aveva assorbito la matrice educativa della pedagogia umanista del Risorgimento europeo sull'educazione e la formazione generale dell'essere umano [...] il quale doveva fagocitare tutte le belle qualità corporee e spirituali» (Hoti, 2002, 144).

La Normale aveva sei classi, tra le quali tre preparatrici, in cui potevano entrare solo allievi che avevano finito una scuola elementare di qualsiasi lingua e, altre tre semplicemente normali. L'educazione e l'apprendimento erano considerati dagli insegnanti della Normale una categoria eterna e complessiva della vita sociale e, il sistema pedagogico si fondava su due idee fondamentali, «l'insegnamento e la conoscenza non hanno confini, valgono per tutti, e l'istruzione è una realtà che si propaga ovunque». L'attività della scuola Normale di Elbasan ha una grande rilevanza storicopedagogica anche per quando riguardano i primi testi albanesi di natura psico-pedagogica indispensabili per la formazione culturale generale dell'insegnante. (Kamani, 1985, 10, 22).

Effettivamente l'educazione per molti secoli è stata considerata come un privilegio di pochi e il processo formativo è stato correlato al potere politico ed economico della società. Per quando riguarda l'educazione universale, soltanto dopo l'avvenuto della rivoluzione industriale diventa urgente applicarla nell'ambito lavorativo, e quindi, la scolarizzazione, ossia l'estensione dell'istruzione sull'intera popolazione, diviene un'esigenza. Quando si mette in moto il processo di democratizzazione dell'educazione:

Le classi sociali sono costrette a limitare gradualmente i privilegi, compreso quello dell'istruzione. Tale cambiamento ha storicamente inizio, almeno in occidente, con la riforma protestante e con la teoria pedagogia di Comenio. Nei secoli XVI 
e XVII incomincia ad affermarsi attraverso le conseguenze prodotte dalla dottrina religiosa e pedagogica di Martin Lutero e della teoria didattica di Comenio il principio dell'educazione universale [...]. II pedagogista polacco sanci con l'espressione "tutto a tutti" il valore enciclopedico del sapere (Boccia, 2001, 114).

L'approccio di Gurakuqi riguardante l'educazione è di natura democratica e può essere analizzato in modo più chiaro prendendo in considerazione i suoi discorsi, le sue lettere e i suoi articoli, dove riesce a elaborare un atteggiamento non solo scientifico ma anche patriottico, in quanto dimostrano una grande conoscitiva cultura:

Da questa scuola saranno lanciati volenterosi apostoli che dirameranno la luce negli angoli più bui del paese e uomini di grande umanesimo, come Orfeo e Anfione, che con la loro saggezza e i loro consigli mitigheranno gli aspri animi degli albanesi, i quali [...] devono sapere che fin dai tempi remoti l'uomo era interessato alla conoscenza e solo [...] dopo la caduta dell'Impero romano, l'istruzione diventò accessibile per tutti finché [...] nel 1378 Vittorino da Feltre comincia a occuparsene dell'istruzione nazionale. In seguito, il suo percorso sarà rintracciato anche da Erasmo da Rotterdam, Lutero, Rabelais da Francia [...] e il grande Kant, Pestalozzi, Friedrich Fröbel, e tanti altri; e nei tempi recenti Erbert Spenser. [...] Nella nostra scuola il cittadino, il contadino e il montanaro conviveranno e diventeranno un corpo solo; qui i Gheghe e i Toschi dimenticheranno i loro nomi; ormai, la lingua albanese, dopo questo indulgente discorso, prenderà un'altra forma e un'altra sembianza: da qui usciranno solo Albanesi, soltanto Albanesi! (Osmani, 136-138).

\section{Nuovi Criteri per Una Nuova Istruzione di Masse}

Per costruire un sistema educativo che passa attraverso le istituzioni scolastiche, occorreva costruire, in primis, determinati criteri e principi teorici. In primo luogo, la scuola doveva essere il luogo per eccellenza dell'educazione nazionale e patriottica, naturale e individuale. Diversi patrioti e intellettuali durante il primo decennio del XX secolo accennavano l'importanza della lettura quotidiana dei libri di carattere nazionalistico e scientifico, legati alla storia, alla geografia e alla lingua albanese. Secondo Xhuvani lo scopo dell'insegnamento e dell'educazione «non è di formare un bambino individualista ma un bambino capace di amare la propria nazione, e per raggiungere questo traguardo l'alunno deve conoscere bene la propria matrice culturale» (Temo, 3 (1982), 95).

Osserviamo che l'autore insiste nella formazione di un individuo responsabile della qualità della vita collettiva, cioè un uomo non individualista che, come sosteneva Tocqueville, «vive per conto suo ed è estraneo al destino di tutti gli altri; i figli e gli amici costituiscono per lui la razza umana; quanto al resto dei concittadini, egli vive al loro fianco ma non li vede; li tocca ma non li sente; non esiste che in se stesso e per se stesso» (Amerio, 2004, 35). Pertanto, quando parlano della libertà, gli uomini del Risorgimento albanese non includono la concezione della libertà moderna, ossia la libertà percepita come un «godimento della propria esistenza», ma l'idea di una libertà antica: «chi non partecipa all'esercizio del potere nella comunità 0 era fuori della comunità oppure non era degno, perciò non era libero» (Ibid., 34).

Xhuvani, essendo uno dei primi teorici della pedagogia albanese, sosteneva che ai problemi dell'educazione della mente, degli scopi educativi, dei doveri e del percorso da seguire per la loro realizzazione, bisognava dare il giusto riguardo scientifico. II nucleo dell'educazione della mente, come il mezzo principale dell'educazione, consisteva «nella comunicazione e nel componimento di tutti i poteri dell'anima». Quello che maggiormente si nota nell'approccio pedagogico di Xhuvani era il bisogno di applicare un «insegnamento profondo, sostenibile e sistematico delle scienze» (Temo, cit., 97). L'autore fu incline a sostenere con irremovibilità il principio dell'autoeducazione e di autoefficacia. Era orientato verso l'applicazione di un insegnamento creativo in cui ogni conoscenza doveva essere l'implicazione di una ricerca e di un'osservazione individuale (Ibid., 98).

In secondo luogo, la scuola doveva sostenere le tradizioni del popolo albanese come l'unica via per formare un'identità nazionale e uno spirito patriottico. D'altronde anche Rousseau redigeva la sua teoria d'educazione moderna basandosi sull'idea che «dobbiamo cercare nella nostra natura e nella nostra storia la formula che deve avere l'ideale educativo, non dobbiamo quindi prendere nulla in prestito dagli altri popoli e dalle altre civiltà» (Barausse, cit., 237). In tutto questo percorso d'apprendimento la lingua nativa era considerata «lo strumento d'insegnamento», mentre i sentimenti e l'elaborazione spirituale «lo strumento per il lancio del carattere di una nazione» (Hoti, cit., 153). In verità «quando parliamo delle nostre scuole», sosteneva Gurakuqi, «dobbiamo intendere solo quelle in cui s'impara l'albanese, per di più, quelle in cui l'apprendimento sia di carattere popolare, basato sulle nostre tradizioni e consono ai bisogni nazionali» (Ibid., 151).

In terzo luogo, la scuola nazionale albanese, minacciata e sopraffatta dalla continua concorrenza e propaganda da parte delle scuole straniere, doveva preservare il suo carattere statale e un certo atteggiamento antagonista nei riguardi dei loro programmi. Lo stesso Gurakuqi sosteneva l'idea che in Albania le scuole private sovvenzionate dagli stranieri dovevano essere addirittura chiuse, giacché essi costituivano soltanto una risorsa di «divisione e denazionalizzazione del 
nostro popolo», anche se, visto da un'altra angolatura, l'atteggiamento politico e ideologico di Gurakuqi sembra in una certa misura contraddittoria, poiché lui stesso aveva finito i propri studi presso il Collegio Severiano. Ma, comunque, Gurakuqi non esclude tutte le scuole straniere: il pericolo della denazionalizzazione proveniva maggiormente dalle scuole greche dove gli albanesi che frequentavano questi ambienti «diventavano più ellenici degli stessi greci» (Ibid., 155).

In ultimo, i patrioti chiedevano la costruzione di un'istruzione nazionale sostenuta da una matrice pedagogica e didattica di grande valore. II modo in cui questo problema sarà trattato da Gurakuqi, Xhuvani, Babë Dudë Karbunara, Hilë Mosi e tanti altri, dimostra l'ampia conoscenza della più importante eredità pedagogica occidentale da parte di questi autori (Ibid., 157).

A proposito del discorso del pensiero pedagogico risorgimentale, si può, inoltre, notare che l'ottica di Xhuvani e Karbunara tende a implementare nel sistema educativo albanese la concezione naturalistica dell'educazione, secondo il quale «non è vero che il fanciullo è cattivo per natura, [...] ma, al contrario, egli porta con sé dalla nascita i germi della virtù sociale, e quindi dobbiamo aiutarlo a svolgere le sue qualità native [...], educarlo secondo le leggi della natura le quali vanno rintracciate nella natura stessa del fanciullo» (Barausse, 2004, 236).

Jorgji Karbunara, conosciuto anche come Babë Dudë Karbunara (Lleshi, 4 (1987), 119) ha costruito il suo pensiero psicopedagogico basandosi sul patrimonio pedagogico di Kostë Berati e tanti altri patrioti albanesi.. Per realizzare i suoi precetti idealistici si doveva riformare non solo l'atteggiamento intellettuale ma anche eseguire una vera e propria innovazione didattica:

Per imparare come si deve una lingua straniera prima bisogna conoscere e padroneggiare la propria lingua e [...] imparare vuol dire studiare sin da piccolo [...]. Già dai sette anni l'essere uomo può apprendere nella stessa misura di un adulto e per questa ragione [...] bisogna stare attenti ai metodi d'insegnamento e ai testi usati (Lleshi, cit., 122-123).

In questo indefesso tentativo di risvegliare l'amore per la patria, l'educazione morale e fisica costituivano parte integrale ed essenziale del programma educativo generale: l'uomo doveva essere ricco di valori morali, sociali e spirituali, ed esso doveva coltivare, attraverso lo studio, una dignità personale e nazionale degna di essere protetta a qualunque costo. Nella sua visione dell'importanza dell'educazione fisica Rousseau evidenziava la funzione morale della ginnastica, in altre parole «allontanare la passione, [poiché] quando le braccia lavorano molto, l'immaginazione si riposa; quando il corpo sta bene, il cuore non si riscalda». Indubbiamente, tanti pedagogisti avevano formulato i loro atteggiamenti teorici sulla questione dell'educazione fisica, la quale, generalmente, doveva «precedere a ogni altra educazione» (Ulmann, 2004, 209-210). L'attività fisica intesa come educazione individuale e collettiva, come strumento di esaltazione della bellezza nazionale e del radicamento delle virtù nazionali, fu il paradigma di Friedrich Ludwig Jahn, il padre fondatore del movimento ginnico tedesco. Secondo Jahn «più grosso è lo stomaco, più vacillante e goffo appare l'uomo, più vuota è la sua anima» (Mosse, 1975, 58).

Tuttavia, la realtà educativa albanese, ereditava parte dell'educazione medievale nel modo di trattare l'allievo, modi che, secondo Karbunara e Xhuvani, dovevano essere percossi da metodi contemporanei d'insegnamento quali «la collaborazione tra maestro e alunno e i discorsi durante le lezioni, capaci di sviluppare le capacità intuitive e pratiche», le quali, come notavamo anche negli atteggiamenti teorici di Gurakuqi, «aiuteranno il fanciullo a trovare da sé il senso delle cose durante le lezioni, i compiti, le passeggiate, i disegni ecc.» (Lleshi, cit., 124-125).

Osserviamo che il pensiero pedagogico di Karbunara segue la scia teorica del grande pedagogista del XVIII secolo Charles Rollin. Egli sosteneva con fermezza che «l'insegnante deve voler bene al proprio alunno e all'insegnamento, deve farsi amare, ma anche rispettare, implementando una forte disciplina che non sia, chiaramente, una critica pubblica 0 un'umiliazione, ma una correzione della cattiva volontà». «Per una formazione positiva», continuava Rollin, «dobbiamo conversare con l'alunno e cercare di convincerlo, [perché] il bambino ha bisogno di sentire che il suo maestro lo ama, usando la parola e anche la ricompensa». Possiamo sostenere che Karbunara riproponeva in un nuovo contesto culturale gli atteggiamenti pedagogici di Pestalozzi: «la costruzione dell'apparato concettuale del bambino deve essere progressivo e sempre affidato al binomio esperienza-intuizione». Per il pedagogista svizzero non era possibile «uno sviluppo completo della persona senza un'adeguata educazione morale, e il raggiungimento morale dovrebbe essere la fine stessa dell'azione educativa della madre e del maestro» (Spadolini, 2007, 273). II pensiero romantico di Pestalozzi sulla pedagogia affiorava dopo il periodo dell'impercettibilità educativa dell'alunno tramandata dal Medioevo in cui «l'educazione concepiva l'educando come un principio di male, e il peccato originale era il germe del fanciullo che bisognava estirpare con la disciplina crudele e la battitura». Secondo questo modello «l'educatore che non punisce l'educando manca al suo dovere, fa il suo male, lo cresce per l'inferno non per il paradiso» (Barausse, cit., 236).

Ed è proprio questo tipo di educazione che Karbunara ripudiava con determinazione. Dalle esperienze pedagogiche dell'insegnante Karbunara «la tradizione albanese ha ereditato una famosa espressione indirizzata agli 
alunni: buona mattina - rugiada rosa. In questo modo, i bambini percepivano l'amore dell'educatore nei loro confronti attraverso gli sguardi, i modi di comunicare e gli atteggiamenti quotidiani» (Lleshi, cit., 124).

\section{Conclusioni}

L'educazione nazionale, come il leitmotiv del nuovo pensiero pedagogico-nazionale, alla luce delle nuove idee europee, proponeva di costruire un sistema educativo che passava attraverso le istituzioni scolastiche, e per realizzarla occorreva costruire, in primis, determinati criteri e principi teorici. In primo luogo, la scuola doveva essere il luogo per eccellenza dell'educazione nazionale e patriottica, naturale e individuale. In secondo luogo, la scuola doveva sostenere le tradizioni del popolo albanese come l'unica via per formare un'identità nazionale e uno spirito patriottico. In terzo luogo, la scuola nazionale albanese, minacciata e sopraffatta dalla continua concorrenza e propaganda da parte delle scuole straniere, doveva preservare il suo carattere statale e un certo atteggiamento antagonista nei riguardi dei loro programmi. In ultimo, i patrioti chiedevano la costruzione di un'istruzione nazionale sostenuta da una matrice pedagogica e didattica di grande valore. II modo in cui questo problema sarà trattato da Gurakuqi, Xhuvani, Babë Dudë Karbunara, Hilë Mosi e tanti altri, dimostra l'ampia conoscenza della più importante eredità pedagogica occidentale da parte di questi autori.

\section{References}

A. Barausse (2004), I maestri all'università: la scuola pedagogica di Roma 1904-1923, Perugia: Morlacchi Editore.

A. Xhuvani 4 (1934), Fjalimi i mbajtur me rastin e 25 vjetorit të Shkollës Normale, Elbasan: "Normalisti".

B. Spadolini (2007), Educazione e società. I processi storico-sociali in Occidente, Roma: Armando Editore.

J. Ulmann (2004), Nel mito di Olimpo. Ginnastica, educazione fisica e sport dall'antichità a oggi, Roma: Armando Editori.

L. G. Mosse (1975), La nazionalizzazione delle masse. Simbolismo politico e movimenti di massa in Germania (1815-1933), Bologna: ॥ Mulino.

Nxënësit e shkollës Normale, "Normalisti", Anniversario 1909-1959, Elbasan.

P. Amerio (2004), Problemi umani in comunità di massa, Torino: Einaudi.

P. Boccia (2001), Sociologia. Teoria, storia metodi e campi di esperienze sociali, Zanichelli: Bologna.

R. Sani (2011), Sub specie educations. Studi e ricerche su istruzione, istruzioni scolastiche e processi culturali e formativi nell'Italia contemporanea, Macerata: EUM.

S. Temo 3 (1982), Aleksandër Xhuvani mbi pedagogjinë dhe edukatën, Tiranë: "Revista Pedagogjike".

Sh. Demiraj, K. Prifti (2004), Kongresi i Manastirit, Tiranë: "Mësonjëtorja".

Sh. Osmani (1998), Reflekse etno-pedagogjike, Tiranë: "Idromeno".

V. Hoti (2002), Luigj Gurakuqi për shkollën shqipe dhe arsimin kombëtar, Shkodër: CP.

V. Kamani (1985), Ndihmesë për historikun dhe traditat pedagogjike të shkollës së mesme pedagogjike "Luigj Gurakuqi", Tiranë: M. Duri. Xh. Leshi 4 (1987), Mendimi pedagogjik i Babë Dudë Karbunares, Tiranë: "Revista Pedagogjike". 
